\title{
TRACCE DI NON FINITO ALLA RICERCA DELLA MORTE (E) DELL'EROE IN CONTESTO KASSENA
}

\author{
Gaetano Mangiameli \\ Università degli Studi di Milano
}

RIASSUNTO: Questo articolo affronta il tema della morte dell'eroe nella cultura kassena dell'Africa occidentale (Ghana e Burkina Faso). La mancanza di dettagli sulla morte dell'individuo eccellente nella mitologia kassena può essere messa in connessione con una cosmologia che concepisce il mondo e la vita come processi sempre in corso e l'azione creatrice degli antenati come perdurante. Se le narrazioni kassena esplorano o incorporano il tema del non finito, questo va inteso non come fatto episodico o accidentale, ma come tratto estetico coerente con un quadro culturale.

PAROLE CHIAVE: morte, eroe, Africa occidentale, kassena, Ghana

ABSTRACT: This article deals with the death of the hero in the Kassena culture of West Africa (Ghana and Burkina Faso). The lack of details about the death of the illustrious individual in Kassena mythology can be interpreted in the context of a cosmology that sees the world and life as neverending processes that are always in the making and the creative actions of the ancestors as persisting. If Kassena narrations explore and embody the idea of the unfinished, this feature is to be interpreted not as incidental, but as an aesthetically congruent cultural element.

KEY-WORDS: Death, Hero, West Africa, Kassena, Ghana 


\section{INTRODUZIONE}

Ogni osservazione etnografica, anche la più microscopica, può essere occasione per stimolare un'ampia comparazione. In antropologia sociale e culturale si è spesso tratto spunto da singoli casi per mettere in discussione le classificazioni esistenti e proporne di nuove, in una tendenza potenzialmente infinita che ha dato luogo a critiche e ripensamenti, basti pensare alle celebri parole di Edmund Leach sulla «raccolta di farfalle». ${ }^{1}$ Nonostante questo rischio, è bene preservare la possibilità di esplorare il contesto della scoperta, oltre a quello della giustificazione, traendo da casi specifici, magari minimi e marginali, come si tenterà di fare in questo saggio, l'ispirazione per successive operazioni comparative da sviluppare in un secondo momento. In questo caso, lo spunto è costituito dalla morte dell'eroe, che sonderemo presso il contesto kassena, in Africa occidentale.

La descrizione della morte può avere l'obiettivo di conferire un senso di compiutezza alla traiettoria di vita dell'eroe. Anzi, invece di essere semplicemente l'ultimo episodio della vita di un individuo eccellente, essa può segnare in effetti una circostanza determinante nel modellare il profilo dell'eroe. Da questo punto di vista, in altre parole, la morte potrebbe costituire una soglia produttrice di senso e permetterebbe la pensabilità stessa del final cut, il "montaggio finale" di una biografia eroica, contribuendo fortemente a dare una forma a questa biografia. In questo articolo, tuttavia, ci soffermeremo su un caso, quello delle narrazioni in circolazione in territorio kassena (Ghana nordorientale e Burkina Faso meridionale), in cui la descrizione della morte, rarefatta se non assente, sembra non suscitare interesse.

In una prospettiva antropologica la morte dell'eroe non è un tema ma una sorta di doppia matrice di variabilità, che può dar luogo a un resoconto in grado di attraversare virtualmente tutte le forme di umanità del presente e del passato per sondare da un lato la morte, dall'altro l'eroe. Per gli scopi di questo articolo, che sono molto moderati rispetto a un obiettivo impressionante quanto la comparazione tra culture sul tema della morte

\footnotetext{
${ }^{1}$ Avvalendosi di classificazioni in tipi e sottotipi, la comparazione, secondo Leach, può giungere a fare di ogni società un sottotipo diverso da ogni altro (si veda LEACH 1973: 15-16). Il rischio che l'individuazione di una proprietà induca a creare appositamente una nuova classificazione eventualmente fine a se stessa, d'altra parte, può essere scongiurato lavorando sulle connessioni o comunque facendo procedere in parallelo lo sguardo sulle differenze e quello sulle analogie.
} 
(e) dell'eroe, si cercherà di rintracciare il contributo di un contesto specifico, quello della cultura kassena dell'Africa occidentale, attingendo alla sfera rituale e al corpus di letteratura orale costituito da miti e leggende. ${ }^{2}$ In antropologia la morte è un tema ampiamente attestato che viene affrontato soprattutto sul piano del trattamento rituale. "Raccontare la morte" o "dare un senso alla morte”, nella lettura dell'antropologia, è innanzitutto ciò che si fa attraverso il rituale funebre, almeno a partire dal contributo fondamentale di Robert Hertz. ${ }^{3}$ Se le società umane affrontano la morte attraverso il rituale, esaminare il rituale funebre significa cogliere il modo in cui una società tematizza la morte, ammesso che ce ne sia uno, per poi magari dover precisare, come avviene nel caso kassena, che la società (o la cultura) presa in esame tematizza a suo modo i morti, più che la morte. In questo le società si avvicinano al lavoro dell'autore di un'opera di finzione, in quanto anche queste producono un testo, in un senso semiotico molto ampio e inclusivo. Così, se un'opera di finzione affronta il tema della morte, lo farà emergendo dalla tematizzazione della morte in un determinato momento storico-culturale e intervenendo su tale tematizzazione. Alla stessa maniera, se, come afferma Marshall Sahlins, «ogni riproduzione della cultura è un'alterazione», ${ }^{4}$ anche il rituale funebre emerge da una tradizione consolidata e nel contempo interviene su questa, ma soprattutto, per gli scopi di questo articolo, e indipendentemente dalla prospettiva diacronica di sviluppo, il rituale funebre, oltre a gestire la morte, è un testo sulla morte. ${ }^{5}$ Qui si vuole porre un segno di (cauta) analogia tra l'opera di finzione che tocca un certo tema e il rituale che gestisce, manipola o coinvolge lo stesso tema. Accostare il rituale o più in generale le pratiche al linguaggio verbale è un'operazione che può facilmente andare incontro a legittimi e doverosi rilievi critici. ${ }^{6}$ In questo caso, tuttavia, non si sta affermando che ciò che l'antropologia vede nel rituale è linguaggio verbale, ma si intende piuttosto procedere in una direzione inversa:

${ }^{2}$ L'autore ha condotto a più riprese prolungati periodi di ricerca sul campo nel Ghana nordorientale a partire dal 2003.

${ }^{3}$ Hertz 1907.

${ }^{4}$ SAHLINS 1986: 126.

${ }^{5}$ Opportunamente, per evocare un classico della letteratura antropologica d'area su questo tema, lo studio di Jack Goody sulle usanze funebri dei LoDagaa del Ghana porta alla presentazione dell'escatologia locale (Goody 1962).

${ }^{6}$ Si veda per esempio JaCKSON 2011 e DesCOLA 2021: 109-131. 
ciò che una società ha da dire sulla morte può essere riscontrato nel rituale funebre, attraverso un'operazione creativa di resa verbale che è tra i compiti dell'antropologia.

Ora, avvicinandoci al tema della morte dell'eroe attraverso il rituale, non solo il testo così concepito non viene registrato come il prodotto del talento creativo di un individuo né dello sforzo consapevole di questi, a differenza di quanto accade con l'opera letteraria di finzione, in quanto il rituale risulta in maniera emergente dalle attività, dal movimento, dalle interazioni, dalla collaborazione e dalle spinte di un collettivo, ma per di più è proprio in relazione a un collettivo, e non soltanto a individui eccezionali, che svolge questa funzione. Se l'antropologia si avvicina al rituale funebre come testo, lo fa pensandolo non solo come testo destinato a tanti (i partecipanti stessi, gli astanti, virtualmente l'intera società), ma anche come testo scritto da tanti (le persone che partecipano al rituale, lo eseguono, lo modificano) e relativo a tanti, cioè alla morte di chiunque. Volendo parlare della morte dell'eroe, questo sembra essere un punto rilevante e problematico: il rituale funebre tratta della morte di chiunque; le regole non scritte (o eventualmente scritte) su tabu, prescrizioni e ogni genere di restrizione o codice di condotta compaiono nella letteratura etnografica, appunto, in relazione alle collettività, alla morte come fatto comune che riguarda innanzitutto le persone comuni, ed è questa una delle ragioni per cui l'etnografia tradizionale viene accusata di omogeneizzare ed essenzializzare società e culture; che ne è, dunque, degli eroi?

A questo proposito, sebbene l'antropologia della morte si applichi ai collettivi e abbia una dimensione inclusiva, coerentemente con l'accezione descrittiva della "cultura", va detto che la descrizione dei rituali funebri e della memoria culturale dei defunti può trovare prototipi significativi nei casi relativi agli individui eccellenti, semplicemente perché spesso queste circostanze permettono di osservare i fenomeni su una scala più imponente, per risonanza dell'evento, partecipazione, investimento simbolico e materiale. È quindi per questa via che ci si può mettere sulle tracce della morte dell'eroe.

Il rituale funebre kassena, in Africa occidentale, si occupa del defunto nel quadro di un idioma dell'abitare. Più precisamente, la gestione della sepoltura nel corso del cosiddetto funerale immediato kassena, il lua lia, esplicita la posizione che il defunto occupa nella storia della casa in cui ha vissuto e in cui sarà sepolto, mentre il funerale finale, lua fulim, si svolge a una distanza variabile di tempo, che può arrivare anche a diversi anni dal 
decesso, segna il passaggio definitivo al mondo degli antenati e contiene elementi di esplicitazione dello status anche se spesso è rivolto a un numero più ampio di defunti, non a uno solo. ${ }^{7}$ Durante il lua lia si trova posto al corpo nel sottosuolo della residenza, scavando una nuova tomba oppure riaprendone una preesistente in base all'identità del defunto. La tomba è assimilata a una sorta di casa nel sottosuolo e dunque nell'aldilà. Un individuo di particolare rilievo, specialmente se si tratta del fondatore della casa, merita una nuova tomba, cioè una nuova stanza, inaugurata appositamente, che in futuro sarà aperta nuovamente per dare sepoltura a persone di prestigio minore. Il segnale chiaro che la cultura kassena ci fornisce attraverso le pratiche di sepoltura è che l'individuo di rilievo è il fondatore di una casa, cioè una persona che può avere compiuto alcune delle seguenti azioni: il fondatore si è sganciato dall'insediamento paterno (la società kassena è patrilineare e virilocale), ha fronteggiato con la forza e con l'astuzia situazioni pericolose o incerte, ha iniziato un nuovo percorso autonomo altrove fondando un insediamento, ha introdotto un nuovo culto o un nuovo principio politico-rituale di organizzazione della vita collettiva. Il fatto è significativo perché ripropone la logica dell'eroe fondatore, migrante e innovatore che si riscontra nei racconti eziologici dei Kassena.

\section{EROI SCISSIONISTI TRA GHANA E BURKINA FASO}

Le narrazioni orali kassena riguardano prevalentemente le origini degli insediamenti, la memoria delle razzie schiavistiche e i rapporti tra umani e non umani nel tempo degli antenati, ${ }^{8}$ e permettono di cogliere il modo in cui i dettagli di microstoria locale si intrecciano con una storia regionale. ${ }^{9}$ Assai diffusa è la figura dell'eroe scissionista, che per una ragione o per l'altra si separa dal gruppo familiare e si mette in viaggio, superando prove rischiose e dando vita a un nuovo insediamento. È questo certamente il caso, tra gli altri, del racconto eziologico di Paga, un chiefdom kassena del Ghana nordorientale, che

\footnotetext{
${ }^{7}$ Si vedano Abasi 1995, CACCiCO - Colombo 1994, Mangiameli 2015.

${ }^{8}$ Cfr. HAHn 2003, Howell 1997, Kibora 2003, Liberski-BAgnOUd 2002, MANGiameli 2016, 2017, 2018 e 2019, TEViu - CALlOW 1976, e ZWERNEMANN 1969.

${ }^{9}$ Cfr. DitTmer 1961, Duperray 1984, Gomgnimbou 2003.
} 


\section{Gaetano Mangiameli}

inizia narrando le avventure di Panlogo, un principe che risiede nel territorio dell'attuale Burkina Faso. ${ }^{10}$ Come vedremo, della morte in questa narrazione si dice ben poco.

Alla morte del capo, il padre di Panlogo, era prevista la competizione tra i figli per la successione. Panlogo partecipò alla competizione, ma fu sconfitto. Egli decise dunque di abbandonare l'insediamento del padre, allontanandosi dalla non meglio precisata località nella quale risiedeva, e di spostarsi con il suo seguito presso Tampela. Qui si fermò per un po' di tempo, ma l'aggravarsi delle tensioni con il fratello vincitore e i suoi sostenitori indusse successivamente Panlogo a cercare di mettersi al sicuro spostandosi ulteriormente. Allontanatisi da Tampela, i fuggitivi trovarono un ostacolo apparentemente insormontabile in un fiume in piena, rischiando dunque di essere raggiunti. Comparve a questo punto un coccodrillo. Panlogo e il coccodrillo si guardarono e si riconobbero: tra loro c'era un'antica relazione che comportava un patto di non aggressione. Fu così rinnovato il patto tra Panlogo e il coccodrillo, o meglio tra le rispettive linee di discendenza: l'uomo giurò che mai i suoi discendenti avrebbero ucciso i coccodrilli, che mai avrebbero fatto loro del male e mai ne avrebbero consumato le carni. Rinnovato il patto, il coccodrillo entrò nel fiume, proprio mentre arrivavano il fratello di Panlogo e i suoi sostenitori sulle tracce dei fuggitivi. Al passaggio del coccodrillo le acque si aprirono, lasciando passare Panlogo e il suo gruppo, e si richiusero subito dopo, bloccando la via agli inseguitori. Archiviata la minaccia, Panlogo raggiunse il luogo nel quale avrebbe fondato una nuova comunità, Kampala.

Dopo un periodo di tempo imprecisato, la popolazione di Kampala era cresciuta considerevolmente di numero ma stava vivendo una fase di decadenza morale. Alcuni abitanti, infatti, violavano il tabu del coccodrillo. Fu in questo contesto che crebbe Naveh, figlio o discendente di Panlogo, ${ }^{11}$ diventando un valente cacciatore. Naveh era solito cacciare liberamente in una vasta area, in un'epoca in cui la zona non era attra-

\footnotetext{
${ }^{10}$ Il racconto eziologico di Paga è stato raccolto sul campo dall'autore (si veda MANGIAMELI 2017: 57-87), che ha anche visionato alcune versioni scritte disponibili localmente, oltre a quella pubblicata da TEVIU CALlow 1976. La discussione sul tema delle variazioni del racconto esula dagli scopi di questo articolo.

${ }^{11}$ Secondo la versione riportata da Awedoba, il padre di Naveh era un principe mamprusi che avrebbe lasciato Nalerigu (nell'attuale Ghana) per stabilirsi a Nawuri (nell'attuale Burkina Faso) e successivamente a Kampala, da cui si mosse poi Naveh prima di stabilirsi a Paga (AwEDOBA 1985: 49). Qui probabilmente vengono attribuiti a un'unica figura, quella del padre di Naveh, eventi riguardanti generazioni differenti di uno stesso segmento lignatico.
} 
versata da confini, per procurare cibo per la sua famiglia. Una mattina, Naveh uscì dal compound di famiglia per andare a cacciare, rinfrescato dall'harmattan, ${ }^{12}$ portando il suo arco e la faretra con le frecce avvelenate. Il suo cane si muoveva con lui mentre avanzava nella foresta. Secondo i programmi, Naveh sarebbe tornato la notte successiva e si sarebbe seduto in compagnia della gente di casa per mangiare tuo zaafe $e^{13}$ e selvaggina fresca. Le cose, tuttavia, non andarono secondo i programmi.

Mentre stava cacciando un'antilope, Naveh la seguì andando sottoterra, all'interno di una buca scavata da un oritteropo. ${ }^{14}$ Spaventato dall'intruso umano, l'oritteropo fuggì dall'entrata principale e la coprì. Naveh restò quindi intrappolato. Accecato dall'oscurità, il cacciatore non riuscì a vedere che anche l'antilope scappava da un'altra uscita. Disperato, si sedette. Intanto, a Kampala, i familiari di Naveh, non avendo notizie, erano a loro volta in preda alla disperazione. Due giorni dopo tornò a casa il cane da caccia, che si era mosso verso Kampala per dare l'allarme.

Allo stremo delle forze, l'uomo giaceva prostrato sul fondo della buca dell'oritteropo, con le labbra screpolate e la pelle slavata, e aveva perso coscienza. Ad assistere a questo c'era un coccodrillo, nascosto in una delle camere dell'estesa buca dell'oritteropo, mentre i parenti di Naveh, avendo visto il cane tornare da solo a Kampala, si erano preoccupati per la sorte del loro congiunto. Nel tentativo di chiarire la situazione, i familiari consultarono un divinatore, il quale disse loro che l'unica maniera per salvare Naveh era ucciderne il cane offrendolo in sacrificio. I parenti eseguirono l'indicazione. Dopo il sacrificio, nella buca dell'oritteropo il coccodrillo strisciò verso Naveh e usò la coda per spazzolare via il sudiciume che aveva sul petto. Naveh si risvegliò e seguì il coccodrillo che gli mostrò una via d'uscita dalla buca e successivamente lo guidò verso il laghetto. Qui, mentre il coccodrillo si accomodava su un argine erboso, Naveh si dissetò e riempì di acqua la sua calabash ${ }^{15}$ per tornare a casa. Una volta arrivato a Kampala, raccontò alla sua famiglia dell'incontro con il coccodrillo e riconfermò il giuramento di Panlogo, secondo il quale nessuno dei suoi discendenti avrebbe ucciso, maltrattato o mangiato coccodrilli,

\footnotetext{
${ }^{12}$ Un vento secco che caratterizza la stagione che va da novembre a marzo.

${ }^{13}$ Una pietanza locale molto diffusa.

${ }^{14}$ Mammifero detto anche 'formichiere africano'.

${ }^{15}$ Il frutto della lagenaria siceraria, altrimenti detta 'zucca a fiasco' o 'zucca bottiglia', viene utilizzato come contenitore per liquidi o alimenti.
} 
ma apprese anche del sacrificio del suo cane. Indignato, decise di abbandonare il suo insediamento originario.

Dopo un lungo cammino, che proseguì per alcuni giorni, Naveh si imbatté in un'antilope e la uccise. Accortosi di essere nelle vicinanze di un luogo abitato, il villaggio di Pindaa, e non potendo trasportare il bottino di caccia da solo, lasciò l'antilope sul posto e andò a cercare qualcuno che gli potesse dare una mano. Quando arrivò nel compound di Pindaa, il padrone di casa chiese alla sua bellissima moglie preferita di dare dell'acqua da bere a Naveh. ${ }^{16}$ La donna, tuttavia, si rifiutò di farlo, dicendo che non avrebbe dato da bere a un essere così spregevole. Un'altra moglie dell'uomo, zoppa e brutta, si alzò allora per andare a prendere dell'acqua da offrire a Naveh. Bevuta l'acqua, il cacciatore chiese e ottenne dal padrone di casa l'aiuto di alcuni uomini per trasportare l'antilope che aveva ucciso. Quando il corpo dell'animale arrivò, l'uomo di Pindaa chiese a Naveh di portarlo nella stanza della moglie bella, ma il cacciatore si oppose e decise piuttosto di depositarlo nella stanza della moglie brutta. Da quel momento, Naveh si stabilì temporaneamente nel compound di Pindaa, contribuendo con il suo bottino quotidiano di caccia.

In seguito, durante la permanenza, Naveh strinse i rapporti con una giovane donna, figlia di Pindaa e della sua seconda moglie. Fu facile per lui ottenere il consenso per il matrimonio, grazie alla simpatia della madre nei suoi confronti. A quel punto, però, Pindaa chiese a Naveh di lasciare la casa e andare a insediarsi altrove con la moglie. Naveh ebbe quindi modo di scegliere liberamente un'area, dato che nei dintorni non viveva nessun altro.

Pindaa informò Naveh che in una certa zona si aggirava un bufalo pericoloso, il gaa nao. Il cacciatore si recò nella zona, trovò il bufalo, lo uccise e poi decise di stabilirsi in quel luogo, pronunciando la frase «A yi paga ywo» ('I miei occhi ammirano questo luogo'), dalla quale deriva il toponimo Paga. Dal bufalo, invece, il nome di Nania ('il posto del bufalo'), il primo nawuuri (sezione genealogico-territoriale) di Paga, cioè l'embrione del chiefdom attuale. Questi eventi si verificarono presso la collina di Kanlaa,

\footnotetext{
${ }^{16} \mathrm{Si}$ tratta di un riferimento alle norme di accoglienza.
} 
primo insediamento di Paga e nome del primogenito di Naveh. ${ }^{17}$ Ebbe origine in queste circostanze anche il kwara, il feticcio del capo supremo, che Naveh ricavò da un corno di gaa nao.

Naveh viveva a Nania e deteneva il kwara, sorto dal suo atto eroico, l'uccisione di gaa nao. Intanto cominciavano ad emergere differenze tra i figli di Naveh. In particolare, solo Kayila, il secondogenito, sembrava essere interessato a occuparsi del kwara. Gli altri figli si tenevano a distanza dal corno, terrorizzati, oppure lo ignoravano, disprezzandone l'effettivo potere. Per un po' di tempo Naveh ricevette le visite di un signore della terra, ${ }^{18}$ Biu, il quale pretendeva da lui offerte per sacrifici annuali, ma non gli rivelava il luogo dei sacrifici. Per qualche anno, dunque, Naveh dipese sul piano rituale da Biu, fino a quando non riuscì a scoprire il luogo in cui questi eseguiva i sacrifici. A quel punto si liberò di Biu e acquisì il controllo sulla terra di tutta Paga.

Morto Naveh, bisognava gestire la successione e quindi anche decidere cosa fare del kwara. Sarebbe spettato al primogenito, Kanlaa, succedere al padre e occuparsi del suo kwara, ma questi non accettò. Decise piuttosto di cedere il kwara a Kayila, il secondogenito, a patto che questi fosse andato a vivere altrove. Kayila, preso il kwara, andò quindi a fondare il suo nuovo insediamento: nacque così la futura sezione di Kayilo. Il primogenito, invece, restò a Nania, vale a dire nei pressi dell'insediamento originario, ma fondando un proprio compound.

Kayila, secondogenito dell'eroe fondatore e detentore del kwara, affidò formalmente il potere a Kum, il terzogenito, che si insediò a sua volta da un'altra parte, fondando anche lui un proprio compound: nacque così Kakuju, quella che oggi è la sezione (nawuuri) del pe, 'il capo'. ${ }^{19}$ Di altri eventuali fratelli, non inscritti in questa genealogia dello spazio, non si sa nulla: le «mnemotecniche dell'abitare ${ }^{20}$ dei Kassena leggono la storia nella rete di rapporti genealogici tra insediamenti.

\footnotetext{
${ }^{17}$ Le denominazioni di alcuni luoghi derivano dai nomi degli antenati che vi hanno vissuto o che vi hanno compiuto atti particolarmente significativi. A questo proposito si può parlare di «uomini che diventano luoghi» (MANGIAMELI 2017: 117-143).

${ }^{18}$ Il signore della terra (tigatu) è generalmente membro di un gruppo di discendenza considerato autoctono con autorità rituale sulla terra. Si veda il par. 3.

${ }^{19}$ Considerato discendente diretto dell'antenato fondatore, il capo rappresenta e incarna l'unità della comunità.

${ }^{20}$ Si veda MANGIAMELI 2017: 117-118.
} 
Kakunu, il luogo originato dal terzogenito Kum e residenza del capo, diventa la sede del potere senza con questo però assumere davvero il controllo effettivo, o quanto meno non del tutto. Alle spalle di Kum e di Kakunu, la fonte di legittimità rimane il secondogenito Kayila con la sua discendenza, cioè l'insediamento di Kayilo. Il nawuuri di Kayilo, da questo momento in poi, è detentore della fonte di riproduzione del potere: Kayila fabbrica una "copia” del kwara di Naveh e la consegna al terzogenito, Kum. L'operazione di produzione e consegna di un esemplare meno potente dell'originale si ripete dopo la morte di ogni capo, nel contesto dell'installazione del successore.

\section{LA MORTE RAREFATTA}

Questa narrazione è inserita in una rete di somiglianze di famiglia che la connette ad altre narrazioni in circolazione in Africa occidentale attraverso confini statali e/o etnici. La figura dell'uomo in fuga dopo la sconfitta nella competizione per la successione al padre è un motivo ricorrente facilmente riconoscibile. Per esempio, nel suo celebre e magistrale lavoro sui Tallensi del nord del Ghana, The dynamics of clanship among the Tallensi, Fortes attribuisce origini mamprusi ai Tallensi, citando a proposito di questi ultimi un resoconto delle origini strutturalmente analogo a quello che riguarda Panlogo, il padre di Naveh. ${ }^{21}$ In figure come quella di Panlogo (e di Naveh) si coglie l'eroe culturale in quanto innovatore, portatore di una istituzione politico-rituale, la paare, epitomizzata dal $k w a r a,{ }^{22}$ che trasforma la realtà sociale del luogo in cui questi si trasferisce.

Panlogo è un uomo sconfitto e in fuga che conserva il carisma necessario a tenere legato a sé un seguito, a fondare un nuovo insediamento e a indicare a tutti le opportune coordinate di comportamento (reiterando il patto con i coccodrilli che ha natura vincolante ai fini dell'appartenenza alla comunità), oltre a mantenere la lucidità e a guidare le operazioni anche nel momento del massimo pericolo. Naveh, dal canto suo, viene

\footnotetext{
${ }^{21}$ Cfr. FORTES 1945: 22.

${ }^{22}$ Le cosiddette autorità neotradizionali attuali, come quelle legate alle varie forme di chieftaincy, cui la paare kassena è riconducibile, nascono dall'intreccio tra potere locale africano e potere coloniale. Sul kwara e la paare kassena si veda DiTTMER 1961.
} 
presentato in maniera più evidentemente connessa alla dimensione dell'azione meritoria, rischiosa e/o dall'esito incerto (si pensi all'offerta dell'antilope e all'uccisione di gaa nao). Entrambi agiscono come eroi culturali innovatori, precisamente in rapporto a un contesto di arrivo che appare spoglio o relativamente arretrato, anche qui coerentemente con un quadro assai diffuso. Nelle narrazioni eziologiche dell'Africa occidentale, alla figura dell'eroe straniero che porta l'innovazione della chieftaincy o del regno, cioè del potere sugli uomini incardinato nel capo o nel sovrano, fa da contraltare la presenza di autoctoni spesso inquadrati come primitivi o preculturali, $i$ quali, a loro volta, incarnano il potere sulla terra proprio della figura del signore della terra. A questa dialettica corrisponde in generale, con dettagli specifici e manifestazioni variabili nelle costituzioni non scritte della regione, l'opposizione tra due linee di discendenza che permangono: quella degli autoctoni, che detengono un'autorità primordiale ed esprimono il signore della terra, e quella degli immigrati, che detengono il potere sugli uomini ed esprimono il capo o il sovrano.

Sebbene l'importanza di personaggi del tipo di Naveh nell'immaginario locale non possa essere in nessun modo sminuita, in quanto si tratta del prototipo stesso dell'individuo eccezionale, non si può non notare, partendo da un sistema di aspettative di altro tipo, il carattere minimale della morte nella narrazione che abbiamo visto. Sia nel caso di Panlogo, sia nel caso di Naveh, infatti, non solo manca una descrizione articolata, esteticamente significativa, di portata drammatica o epica, della morte di Panlogo o di Naveh, ma il momento del decesso viene addirittura dato per scontato e archiviato senza alcun dettaglio. Se il narratore non ci dice come siano morti i due personaggi, è il caso di riflettere su questa omissione.

L'assenza di un "approfondimento" sul tema della morte dell'eroe non può essere sottoposta a critica, ma va acquisita in quanto tale. L'episodio della morte è irrilevante dal punto di vista della cultura kassena, che elabora la memoria dell'eroe prescindendo del tutto dalle dinamiche che hanno portato alla morte. In contesto kassena la figura dell'antenato fondatore viene commemorata attraverso la sua trasposizione spaziale, che va letta nella logica per cui gli uomini diventano luoghi. L'antenato ha dato il via alla fruizione di uno spazio inaugurando un compound dal quale sono nati successivamente ulteriori insediamenti "figli" (il rapporto di filiazione tra insediamenti è consapevolmente ed espli- 
citamente richiamato dai membri di ciascun gruppo di discendenza): gli antenati diventano i luoghi abitati cui hanno dato vita; spesso essi danno il loro nome a quei luoghi e alla gente che vi abita; il luogo della sepoltura, che coincide con lo spazio abitato, ospita anche le operazioni rituali rivolte agli antenati stessi.

L'eroe fornisce un modello, per quanto rarefatto e privo di dimensioni psicologiche: un uomo eccellente (1) in generale ha successo nella caccia; (2) porta a termine imprese rischiose, quali liberare un territorio dalla minaccia di un animale pericoloso ( $\mathrm{gaa}$ nao); (3) ha la forza e l'autonomia per sganciarsi dall'insediamento paterno e fondarne uno nuovo; (4) innova la vita collettiva regolandola attraverso la paare, che irrompe nello scenario locale attraverso il feticcio $k w a r a$, a sua volta ricavato dall'impresa eroica (si tratta di un corno di gaa nao); (5) ha una discendenza ampia che cresce e si dirama dopo la sua morte. Ora, mentre i punti (1) e (2), per certi versi anche il (4), possono incarnare un modello che in quanto tale può essere reso eterno nella sua unicità e in qualche modo imitato, i punti (3) e (5), che peraltro sono strettamente connessi tra loro, sono la chiave d'accesso a una logica generativa che ha un'importanza cruciale.

Richiamando una formula assai efficace, usata durante un colloquio da un anziano kassena mentre si discuteva di Naveh e in particolare di quanti e quali fossero i suoi figli, «I nawuura sono i navebia», cioè le sezioni genealogico-territoriali (nawuura) sono i figli di Naveh (navebia). ${ }^{23}$ L'anziano aveva premesso che non c'era modo di sapere quanti fossero e come si chiamassero i figli dell'eroe fondatore. Dal punto di vista locale, l'unico supporto della memoria della discendenza di Naveh non poteva che essere la mappa genealogica stessa: ciò che un uomo lascia con la morte è la casa che ha fondato; ciò cui lo stesso uomo dà vita, tuttavia, non corrisponde alla fotografia dell'esistente al momento della sua morte, ma cresce con il passare del tempo. I meccanismi della scissione, dell'allontanamento e della fondazione di nuovi insediamenti si replicano all'infinito in una serie di filamenti che si prolungano e si diramano, anche se a volte alcuni dei filamenti stessi prendono la via del declino, interrompono la loro crescita e poi si spengono. Evidentemente questo processo può essere apprezzato solo in corso d'opera e non è mai concluso. Questo dato è estremamente significativo perché permette di comprendere come una cultura possa decidere di preservare e celebrare non tanto un oggetto dotato di

${ }^{23}$ Kakuyu (Paga), Upper East Region, Ghana, marzo 2009. 
valore simbolico (come un monumento) quanto una logica generativa: l'importanza dell'eroe fondatore è in costruzione qui e ora.

Di fronte al sokwio, l'insediamento principale di una sezione territoriale, si trova un altare di forma circolare, il nabaari, presso il quale vengono eseguite le offerte sacrificali rivolte agli antenati. La presenza del nabaari (letteralmente 'prima-uomo', cioè 'antenato') segnala il carattere primordiale e fondativo dell'insediamento rispetto agli altri della stessa sezione genealogico-territoriale: qui si è mosso l'antenato, che ha dato vita a questo compound; qui si colloca l'origine degli altri compound della sezione, i figli; qui l'antenato è stato sepolto, tanto che il nabaari può essere considerato anche un indicatore di sepoltura importante. Tuttavia, le vicende altalenanti della vita possono portare un sokwio a essere abbandonato ed eventualmente sostituito da un altro compound, nel quale verrà eretto anche un nabaari. La finzione genealogica permetterà progressivamente di considerare quel nuovo luogo il sokwio, cioè l'insediamento anziano, anche se questo è materialmente falso. Dunque, sebbene il nabaari segnali l'anteriorità genealogica dell'insediamento, per cui il sokwio è genitore degli altri compound, si potrebbe trattare di una finzione. Per i nostri scopi, in questa sede, si tratterebbe quindi di riconoscere come la stessa sepoltura non segnali la fine, ma un inizio. Questo spunto si va ad aggiungere alla consapevolezza di quanto neanche la morte sia configurabile davvero come occasione per il final cut. Il ruolo marginale o azzerato che riveste nell'economia della narrazione la modalità attraverso la quale l'eroe trova la morte va compreso tenendo conto che nel contesto locale il senso della vita e l'efficacia delle azioni del personaggio in questione devono necessariamente dispiegarsi nel tempo dopo la sua morte.

\section{CHI È L'EROE?}

In una sorta di tentativo di mitopoiesi esogena, proviamo adesso ad accostare al mito di fondazione di Paga un racconto che sembra essere collegato a questo, seppure narrato da un punto di vista differente, che non dà luogo a una variante, ma piuttosto sembra raccontare una parte complementare dell'intreccio, una parte omessa dalle storie di cacciatori che prevalgono su pericolosi bufali: la stessa storia, ma raccontata dal punto di vista 
dei bufali. Questa storia, "La moglie del bufalo", circola in Africa occidentale, per esempio presso i Kassena, come narrazione derivata dal tempo delle origini, ${ }^{24}$ ma è imparentata anche con il complesso mitico-narrativo del Bagre, che fu raccolto da Jack Goody presso i LoDagaa nel Ghana nord-occidentale. ${ }^{25} \mathrm{Si}$ narra di un bufalo fortissimo e pericoloso, della cui presenza nel territorio gli uomini non riescono a venire a capo, esattamente come nel caso di gaa nao, che viene poi ucciso da Naveh. Il protagonista della storia sembra essere il bufalo, che ha una moglie incinta e si aggira nella foresta per procurare cibo per la famiglia, a maggior ragione dopo la nascita del figlio. Durante una delle sortite del bufalo nella foresta alla ricerca di cibo, entra in scena un cacciatore particolarmente dotato, in possesso di alcune magie che rendono prodigiosa la sua attività venatoria. Il cacciatore si nasconde in attesa del momento giusto per colpire, il bufalo sente un rumore e si guarda intorno, ma il cacciatore spara e lo colpisce. Dopo essere stato colpito, il bufalo non muore, ma chiede «Chi è?»; il cacciatore si fa avanti e si mostra all'animale, dicendo «Sono io». A quel punto l'animale parte alla carica contro il cacciatore, il quale però lancia in alto il fucile e poi vola per andarsi a sedere sul fucile, restando così sospeso in aria. Da quella posizione sicura, egli guarda il bufalo impotente. L'animale, guardando il cacciatore seduto in aria, gli dice: «Dicevi di non saper volare». Il cacciatore gli risponde: «Tu dicevi di non saper parlare». Il bufalo cade a terra, morto.

Successivamente la moglie del bufalo scopre che il marito è stato ucciso e parla al figlio: «Tuo padre è stato ucciso. Dovrò sforzarmi e soffrire per occuparmi di te da sola, ma troverò chi è stato. Quando sarai cresciuto e ti avrò lasciato andare in giro da solo a procurarti il cibo, io andrò a cercare chi ha ucciso tuo padre». Effettivamente, quando giunge il momento, lascia il figlio e si muove per realizzare la promessa di vendetta. Lungo la strada si trasforma in una bellissima donna e si indirizza verso l'insediamento umano, più precisamente verso il mercato, decisa a incontrare il cacciatore, a farlo innamorare e a sposarlo per poi ucciderlo.

La prima parte del piano si realizza velocemente, ma il padre del cacciatore, che è la persona che ha dato al figlio i poteri magici che quest'ultimo usa nelle battute di caccia, è in grado di vedere che sua nuora è la moglie del bufalo e capisce che è venuta per ucci-

\footnotetext{
${ }^{24} \mathrm{Si}$ veda Mangiameli 2019.

${ }^{25}$ GOODY 1972.
} 
derlo. Nel corso della prima notte dopo le nozze, che gli sposi trascorrono sul tetto di una stanza, la moglie induce il marito a raccontarle i suoi segreti, mentre il padre del cacciatore, nascosto, ascolta:

La moglie disse al cacciatore: «Sei forte, ma non puoi darmi tutto quello che voglio». Il cacciatore chiese: «Che cosa vuoi?».

Moglie: «Tutto quello che usi per essere forte, lo voglio anch'io».

Cacciatore: «Se vado nella foresta e trovo un animale forte che voglio prendere, gli sparo. Poi, se cerca di reagire e mi attacca, io lancio il mio fucile molto in alto, poi vado su anch'io e mi ci siedo sopra».

M: «E se io volo in alto e vengo a prenderti, tu che fai?».

$\mathrm{C}: \ll$ Mi trasformo in erba».

$\mathrm{M}$ : «E che fai se l'animale si mette sull'erba e la distrugge?».

$\mathrm{C}: \ll \mathrm{Mi}$ trasformo in un arbusto».

$\mathrm{M}: \ll$ E se l'animale rompe anche quello?».

$\mathrm{C}: \ll$ Mi trasformo in un albero».

$\mathrm{M}: \ll$ E se distrugge l'albero?».

Il cacciatore stava per rispondere che in quel caso si sarebbe trasformato in un ago (garilwe), ma non fece in tempo a completare la frase per l'intervento del padre, il quale stava ascoltando e lo interruppe: «Idiota, non dirlo!». ${ }^{26}$

Il cacciatore, interrotto dal padre, non arriva a pronunciare la parola garilwe ('ago'), ma solo gari. La moglie si chiede che cosa significhi quella parola, che non conosce. Il giorno dopo la donna propone al cacciatore una visita alla famiglia di lei. Prima della loro partenza, il padre parla con il cacciatore. E lo mette in guardia. Una volta nella foresta, dopo un certo percorso, la donna si isola per un attimo, si trasforma nuovamente in bufala e attacca il marito. Questi non ha con sé il fucile, quindi non può volare; si trasforma in erba; la bufala distrugge l'erba e poi rivela la propria identità; il cacciatore si trasforma in un arbusto, che viene travolto dalla bufala, poi in albero, che a sua volta viene distrutto dall'animale, infine in ago, che va a infilarsi nella coda della bufala. L'animale si muove ripetendo la parola gari fino a quando l'ago non cade per terra, senza che lei se ne accorga.

${ }^{26}$ MANGIAMELI 2019: 162. 
A quel punto il padre del cacciatore usa i suoi poteri per trarre in salvo il figlio, riportando l'ago a casa e poi trasformandolo nuovamente in uomo. A quel punto gli dice «Te l'avevo detto».

La storia de "La moglie del bufalo" o, se si preferisce, de "La moglie del cacciatore", riveste un interesse particolare se accostata al mito di fondazione incentrato sulla figura di Naveh o comunque al motivo ricorrente del cacciatore/migrante/innovatore. L'intreccio potrebbe essere compatibile con quello del mito di fondazione, ma la vicenda di Naveh è evidentemente narrata dal punto di vista degli umani, per quanto i non umani abbiano già un ruolo importante, non di mero contorno, e siano soggetti attivi, mentre ne "La moglie del bufalo" l'equilibrio tra le parti rende ambiguo l'orientamento complessivo. Chi è l'eroe, qui, ammesso che ce ne sia uno? Nell'ambiguità di fondo sembra di poter individuare i protagonisti nei bufali e di potere scorgere semmai negli umani, e in particolare nel cacciatore, gli antagonisti. Il cacciatore elimina un animale pericoloso e lo fa con strabilianti prodigi, ma non sembra che il narratore voglia tributargli l'onore dello status eroico. Il successo nella caccia è il risultato del possesso di magie e sortilegi elargiti dal padre, mentre l'uomo non è dotato di particolari qualità e si dimostra piuttosto ingenuo al cospetto della moglie. Chi si muove autonomamente per un'impresa rischiosa e ad alta tensione ideale, quella di mantenere la promessa di vendicare la morte del marito, andando a inoltrarsi nel territorio degli umani per affrontare l'avversario non in uno scontro ad armi pari ma con la minaccia ulteriore della magia, infatti, è la bufala. Questo episodio narrativo sul mondo delle origini mette in scena un doppio smarcamento: l'eroe, infatti, non è maschio e non è umano.

L'unico personaggio che effettivamente muore è il bufalo. La morte dell'animale ha una grande efficacia scenica: colpito dal cacciatore, il bufalo non muore subito, ma solo dopo l'esplicitazione di uno scarto tra piani ontologici: «Avevi detto di non saper volare», dice il bufalo al cacciatore, ricordando a quest'ultimo i limiti corporei dell'umanità nel mondo in cui il bufalo pensa di vivere; «Avevi detto di non saper parlare», gli risponde l'umano, sottolineando, con un paradosso, che il bufalo deve aver superato i confini tra mondi differenti fatti di prospettive incorporate differenti e di possibilità differenti. Nella tendenziale simmetria di questa narrazione, l'uomo rischia nell'affrontare il bufalo così come il bufalo rischia nell'affrontare l'uomo. Il bufalo, tuttavia, fa 
qualcosa in più mettendo in luce gli scarti di realtà, dimostrandosi così un coraggioso esploratore di domini ontologici altri.

\section{LA MORTE E L’ESTETICA DEL NON FINITO}

Per quanto la sequenza narrativa finale de "La moglie del bufalo" abbia la sua conclusione, il motivo di fondo resta aperto. La bufala ha cercato la vendetta, il padre del cacciatore è intervenuto a difesa del figlio, il cacciatore torna a casa, ma tutti e tre $\mathrm{i}$ personaggi sono vivi. Ogni sviluppo ulteriore è ancora possibile. Il narratore, tuttavia, si ferma qui. Nel mondo della produzione industriale di finzione, il finale aperto è uno stratagemma ampiamente utilizzato per lasciare spazio a nuovi episodi e dunque a ulteriori introiti. Ora, non si può certo escludere, nel caso delle narrazioni orali, che un finale sospeso possa avere a che fare con il piacere di continuare a raccontare in un secondo momento, ma sembra qui più opportuno accogliere come culturalmente emblematica questa immagine della narrazione che si ferma quando i personaggi hanno traiettorie biografiche ancora in corso d'opera.

Sembra di poter cogliere in questo un suggerimento a sottolineare l'importanza del non finito, non come fatto episodico o accidentale, come errore, imprecisione o dimostrazione di sciatteria, ma come tratto estetico coerente con un quadro culturale. In particolare, si può opportunamente pensare di attraversare le culture leggendo il rapporto variabile di attrazione/repulsione tra la trattazione del tema della morte e il non finito. Su questo punto, l'esame delle cosmologie africane può fornire un ricco panorama di materiale etnologico.

Più precisamente, nei racconti eziologici kassena è molto frequente una caratterizzazione dell'eroe in quanto fondatore. Il fondatore è un innovatore in movimento, del quale si narra come è emerso, da dove è arrivato e/o in che modo ha dato forma a un territorio o a un abitato, avviato un'istituzione, dato vita a una formazione sociale, inaugurato un culto. Altrettanto frequente è il trattamento minimale riservato alla morte dell'eroe. Gli sviluppi successivi agli eventi originari che coinvolgono i fondatori sono 
spesso non precisati, ma lasciati aperti, coerentemente con prospettive socioculturali caratterizzate da una significativa disponibilità all'indeterminato.

Può dunque emergere da queste riflessioni la possibilità di cogliere un parallelismo tra un ridotto interesse per la morte, che si coglie nella trattazione laconica del tema, e la valorizzazione dell'incompiuto, che si può leggere nella proliferazione di pratiche e narrazioni che lasciano grande spazio a processi in corso, a rimodellamenti, cambi di direzione e risemantizzazioni, dove una certa enfasi sulla tradizione camuffa a malapena un'idea di cultura fondamentalmente mobile, negoziabile, in continua trasformazione.

Una prospettiva comparativa su questi temi può opportunamente richiamare e valorizzare l'idea secondo cui alcune società «hanno cura dei morti, non della morte». ${ }^{27}$ Se la memoria dei morti può trovare spazio in vari aspetti della sfera rituale e della cosmologia, e la presenza dei morti si può esprimere attraverso il perdurante dipanarsi dei processi socioculturali da loro attivati, aggiungendo profondità alle rispettive biografie, in particolare quando si tratta di individui eccellenti, un concetto astratto di morte non attrae necessariamente la stessa attenzione, così come la descrizione della morte dell'eroe non acquisisce necessariamente funzionalità in relazione al modellamento del personaggio.

Giungendo alle conclusioni, può essere opportuno richiamare un elemento che riveste un ruolo di primo piano nella cultura kassena, nonché nel panorama della savana antropizzata in cui la società kassena abita: il puru, un centro rituale per certi versi enigmatico che si trova nei pressi dell'ingresso delle abitazioni. Si tratta di un cumulo nel quale ogni giorno vengono ammassati il terriccio e i rifiuti raccolti nelle operazioni di pulizia del mattino e nel quale vengono interrate anfore contenenti placenta in occasione di ogni parto avvenuto in casa. Esso può assumere dimensioni notevoli o quasi impercettibili, in base all'intensità della vita che si svolge nell'abitazione: un compound grande e densamente abitato avrà necessariamente un puru più grande rispetto a un piccolo insediamento o a una dimora in via di abbandono. Esattamente come le vicende dell'insediamento inaugurato dal fondatore proseguono dopo la sua morte ma sono comunque collegate al suo atto iniziale, l'andamento materiale del puru rende visibile l'eventuale

\section{${ }^{27}$ MACHO 2002: 960.}


successo dell'atto fondativo mentre esso è in corso, incarna i processi di vita sociale dell'insediamento nel loro farsi, senza una direzione prevedibile e senza una conclusione. Il puru è un oggetto costruito collettivamente e socialmente centrale ma mai completo, che attira l'attenzione sulla prospettiva di lunga durata e sul potenziale. In quanto tale, esso accenna allo stesso tema cui ci siamo accostati in questo articolo. Con il contributo del puru, infatti, possiamo leggere più agevolmente il disinteresse per la morte che abbiamo intravisto nelle narrazioni kassena: esso va inteso non all'insegna della mancanza, ma come apertura alla potenzialità. 


\section{BIBLIOGRAFIA}

ABASI 1995 = Augustine Abasi, Lua-lia, the 'fresh funeral': founding a house for the deceased among the Kasena of north-east Ghana, in «Africa», 65, 3 (1995), 448475.

AWEDOBA 1985 = Albert Awedoba, Aspects of Wealth and Exchange with Reference to the Kasena-Nankana of Ghana, Tesi di dottorato, Università di Oxford, 1985.

CACCICO - COLOMBO 1994 = Giuliana Caccico - Enzo Colombo, Cerimonie funebri Kasena: L'incenerimento della faretra, in «Africa. Rivista trimestrale di studi e documentazione dell'Istituto Italo-Africano», XLIX (1994), 85-95.

DESCOLA 2021 = Philippe Descola, Oltre natura e cultura, Milano, Cortina, 2021.

DITTMER 1961 = Kunz Dittmer, Die sakralen Häuptlinge der Gurunsi im ObervoltaGebiet Westafrica, Hamburg, Cram, De Gruyter und Co, 1961.

DUPERRAY 1984 = Annemarie Duperray, Les Gourounsi de Haute-Volta, Stuttgart, Steiner, 1984.

FORTES 1945 = Meyer Fortes, The dynamics of clanshipamong the Tallensi, eing the first part of an analysis of the social structure of a Trans-Volta tribe, London, Oxford University Press, 1945.

GOMGNIMBOU 2003 = Moustapha Gomgnimbou, Les sources de l'histoire du peuplement du pays kasena, in Histoire du peuplement et relations interetbniques au Burkina Faso, éds. Richard Kuba - Carola Lentz - Claude Nurukyor Somda, Paris, Karthala, 2003, 259-273.

GOODY 1962 = Jack Goody, Death, property and the ancestors. A study of the mortuary customs of the LoDagaa of West Africa, Stanford, Stanford University Press, 1962.

GOODY 1972 = Jack Goody, The myth of Bagre, Oxford, Clarendon Press, 1972.

HAHN 2003 = Hans Peter Hahn, A propos d'une histoire régionale des Kassena au Burkina Faso, in Burkina Faso. Cent ans d'histoire, 1895-1995, sous la direction 
de Yénouyaba Georges Madiega - Oumarou Nao, Paris, Karthala, 2003, 14311441.

HERTZ 1907 = Robert Hertz, Contribution à une étude sur la représentaton collective de la mort, in «L'Année sociologique», X (1907), 48-137.

HOWELL 1997 = Allison Howell, The religious itinerary of a Ghanaian people. The Kasena and the christian gospel, Frankfurt am Main, Peter Lang, 1997.

JACKSON 2011 = Michael Jackson, La conoscenza del corpo, in «Molimo. Quaderni di Antropologia culturale ed Etnomusicologia» - L'esperienza del corpo, a cura di Lorenzo Ferrarini, 6 (2011), 43-74.

KIBORA 2003 = Ludovic Kibora, Mythes d'origine comme fondement du peuplement et de l'organisation sociale du village kasena de Kaya-Navio, in Histoire du peuplement et relations interethniques au Burkina Faso, éds. Richard Kuba - Carola Lentz Claude Nurukyor Somda, Paris, Karthala, 2003, 275-288.

LEACH 1973 = Edmund Leach, Nuove vie dell'antropologia, Milano, il Saggiatore, 1973 [ed. or. 1961].

LIBERSKI-BAGNOUD 2002 = Danouta Liberski-Bagnoud, Les dieux $d u$ territoire. Penser autrement la généalogie, Paris, CNRS Editions, 2002.

MACHO 2002 = Thomas Macho, Morte, in Le idee dell'antropologia, a cura di Christoph Wulf, Milano, Bruno Mondadori, 2002, 960-976.

MANGIAMELI 2015 = Gaetano Mangiameli, La densità di una transizione. Successione, divinazione e infanticidio nel Ghana nordorientale, in «Antropologia e teatro», VI (2015), 110-139.

MANGIAMELI 2016 = Gaetano Mangiameli, L'invenzione precoloniale dei Gurunsi. Le razzie schiaviste e la genesi di un etnonimo in Africa occidentale, in «L'Uomo Società Tradizione Sviluppo», 1 (2016), 57-75.

MANGIAMELI 2017 = Gaetano Mangiameli, L'origine plurale. Miti di fondazione kassena, Milano, Cortina, 2017.

MANGIAMELI 2018 = Gaetano Mangiameli, Miti di fondazione e cronologie in Africa occidentale. Elementi di storia locale kassena, in «Antropologia e teatro», IX (2018), 17-32. 
MANGIAMELI 2019 = Gaetano Mangiameli, Il teatro degli antenati. Note su Lévi-Strausse la mitologia kassena, in «Antropologia e teatro», Numero speciale 2019, 154-169. SAHLINS 1986 = Marshall Sahlins, Isole di storia, Torino, Einaudi, 1986.

TEviU - CALlow 1976 = Adjevu Teviu - John Callow, The founding of Paga, Accra, Institute of African Studies, 1976.

ZWERNEMANN 1969 = Jürgen Zwernemann, La fondation de Pô: Essai d'interpretation des traditions orales d'une ville kasena, in «Notes et documents voltaïques», II, 2(1969), 3-15. 